\title{
PROGRESS ON MODELING OF ULTRAFAST X-RAY STREAK CAMERAS *
}

\author{
G. Huang, J. M. Byrd, J. Feng, J. Qiang, W. Wan, LBNL, Berkeley, CA 94720, U.S.A.
}

\begin{abstract}
Streak cameras continue to be useful tools for studying phenomena on the picoseconds time scale. We have employed accelerator modeling tools to understand and possibly improve the time resolution of present and future streak cameras. This effort has resulted in an end-to-end model of the camera. This model has contributed to the recent measurement of $230 \mathrm{fsec}$ (FWHM) resolution measured at $266 \mathrm{~nm}$ in the Advanced Light Source Streak Camera Laboratory. We describe results from this model that show agreement with the experiments. We also extrapolate the performance of this camera including several possible improvements.
\end{abstract}

\section{INTRODUCTION}

Streak camera is one of the major diagnostic tools for the ultrafast phenomenon. The products with sub-picoseconds temporal resolution for visual light and about one picoseconds for $\mathrm{x}$-ray are already commercial available [1]. Laboratories have reported even higher temporal resolutions [2,3]. In order to develop streak camera with even higher temporal resolution, we build an end-to-end model by the accelerator modeling tools MAFIA. The interaction between electron bunch and electric-magnetic field are analyzed. The simulation results agree well with the experiments. Following analyses of current meander-line based streak camera, a new novel design using deflecting cavity are proposed. Preliminary simulation results are shown here.

\section{MODELING CURRENT CAMERA}

\section{ALS streak camera model}

ALS has developed streak camera for years and the homemade streak camera is used in beam line [4]. The modeling work started from the current streak camera, which consist a photo cathode and grid anode, a pair of meander plane deflector, a solenoid acting as lens and a photoconductive switch.

The principal of the streak camera can be described as following: When the laser comes and triggers the photo cathode, the photoconductive switch is also triggered. Then photo cathode generates the electron bunch containing the timing information of the laser pulse. The switch exports a step function pulse with limited rising time to drive the meander line. The electrons are accelerated in the anode-cathode gap and drift a while to the entrance of the meander plane. The pulse also

*Work supported by the Office of Science, U. S. Department of Energy, under Contract No. DE-AC02-05CH11231

\#ghuang@lbl.gov propagates to the entrance of the meander plane with right bias. The meander plane propagate quasi-TEM mode and the longitudinal phase velocity are slowed down by the

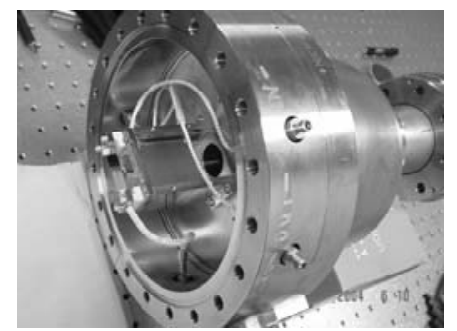

a)

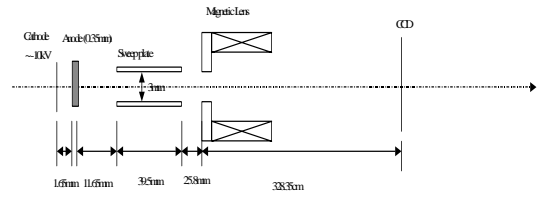

b)

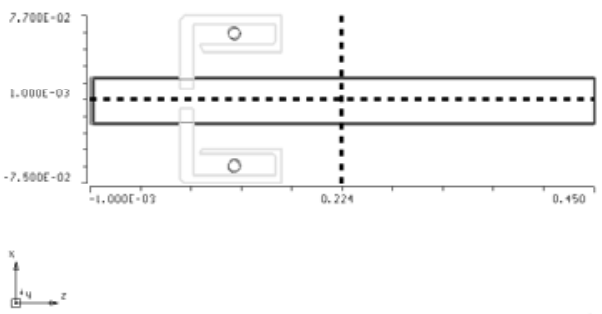

c)

Figure 1 a) Picture of ALS Streak Camera (ALSSC)

b) Block diagram of ALSSC

c) MAFIA model of ALSSC

meander structure. The electron bunches are deflected by the field between the two meander plane, so that the time modulation is converted to the transverse (vertical) momentum modulation. And the bunch is then focused to the image plane and finally monitored by the monitor (either MCP or CCD). Figure 1.a is a picture of the ALS streak camera and 1.b is the block diagram of that camera.

The EM field in the streak camera consist static part and dynamic part. The acceleration field and the solenoid field are the static image system. The meander plane is the dynamic component of the system, the sweep pulse propagate through it, meantime a DC bias is used to pull the image back to the center of the CCD. The static part is a solenoid base imaging system and is designed by COSY code.[5] To model the dynamic part of the camera, we must have an EM solver, which should be able to deal the complex geometry and material as well as tracking electron in that EM field. We build an end-to-end model 
of streak camera in MAFIA included all components and fields except space charge effect.

\section{Simulation and experiment results comparison}

Since the streak camera are integrated in the vacuum, most of the intermediate results of the streak camera are not measurable or even accessible. We have compared following parameters with the simulations: the TDR result of the meander plane, the static image size, the bias sensitivity and the dynamic measurement result including the sweep speed, and the temporal resolution. Beside single run comparison, the systematic parameter sweep is also progressing.

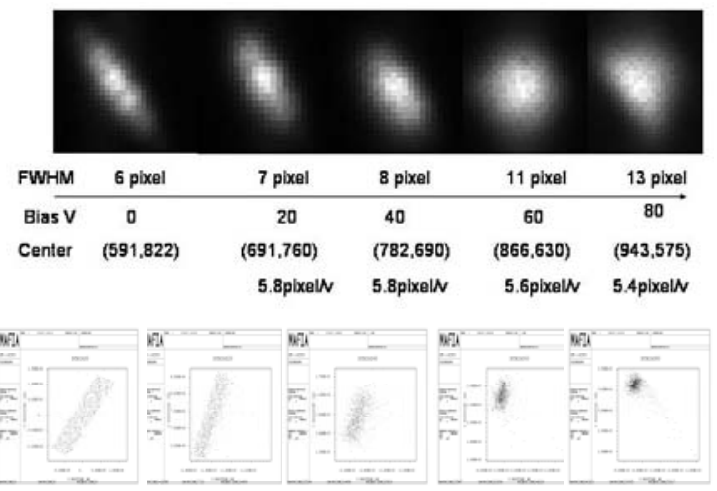

Figure 2 Comparison of the sweep speed from measurement (top) and simulation (bottom)

Figure 2 is the experiment and simulation result of the bias sensitivity. The image spot size increase because of the imperfect lens field, which hints the good field range of the static imaging system.

\begin{tabular}{|c|c|c|}
\hline \multirow{2}{*}{$\begin{array}{c}\text { De deflection } \\
\text { voltage }\end{array}$} & \multicolumn{2}{|c|}{ Bias sensitivity (um/V) } \\
\cline { 2 - 3 } & Experiment & Simulation \\
\hline 20 & 76.5 & 74.5 \\
\hline 40 & 75.5 & 73 \\
\hline 60 & 70.3 & 70.3 \\
\hline 80 & 66.5 & 66.5 \\
\hline
\end{tabular}

Figure 3 shows the experiment and simulation result of two bunches separated by 500fs. Two peak can be resolved clearly and the distance between them are all 7
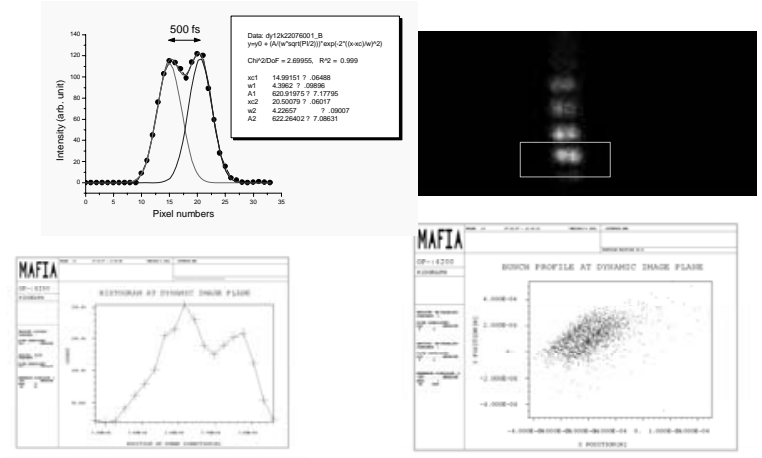

Figure 3 Comparison of dynamic experiment (top) and simulation (bottom) results of images at image plane (right) and it's histogram in sweep direction (left) pixels.

The agreement between the simulations and experiments shows the availability of the end-to-end model. And then the model can be used to research the physics in the streak camera.

\section{Electron bunches interact with the EM field in the streak camera}

After the electrons are generated from the cathode, the electric field accelerates them to a relative high energy (typically about $10 \mathrm{keV}$ ). The energy spread at the very beginning generates the major part of the temporal resolution. This transit time dispersion term (in acceleration field) is inverse proportional to the acceleration field.

After the electrons enter the meander plane, the electric field force them go on a parabolic path. At the exit of the meander plane, the effect of the kick can be back track to the cathode. By comparing the virtual object on the cathode with the size of the electron, we can analyses the temporal resolution and the sweep speed at the cathode. If multiply the sweep speed at the cathode with the static magnification of the image system, we can derive the sweep speed at the image plane.

In ideal case, the electron is synchronized with the slowed wave in the meander plane. The electron bunch size is small enough compare to the field aberration of the meander plane and the solenoid. The slope of the input pulse can be reserved during propagation. So for a step function with linear ramp, the difference between two bunches with a given time separation is constant. But in real world, none of the above is true [6].

The meander line structure has intrinsic cutoff frequency related to the length of the meander leg. The fast rising pulse is deformed by this cutoff and the forward wave is added up with the reflect wave. The longitudinal phase velocity is not a constant any more. The electron velocity cannot match the "pulse" velocity and the overall result is the electron slipping on the pulse. Then the kick taken by the electron is an integral of the force while it slips relative to the pulse.

The limit size of the bunch in the meander plane causes both longitudinal and transverse effect. Longitudinally, a correlation between the transverse position and energy spread is added to the bunch and lengthen bunches while propagating in the deflector. Transversely, fringe field acting on bunches cause defocus on the image.

\section{Proposing deflecting cavity based streak camera}

The development of meander line based streak is limited by its bandwidth and field distribution. Deflecting cavity based streak camera is proposed.

Cavity running on TM110 mode is proposed as crab cavity in the Collider and light source $[7,8]$. Most of them are talking about SC cavity for low power with high deflecting voltage. For the application in the streak camera, we consider the system simplicity and relative high deflecting voltage. So we select normal conducting cavity with solid-state power amplifier. 
Figure 4 a) shows the geometry of a simplified model of the deflecting cavity in the proposed streak camera. The two degenerate TM110 mode are separated by two metal poles which is much simple then the squash cavity shape. Figure 4 b) is the magnetic field distribution of that

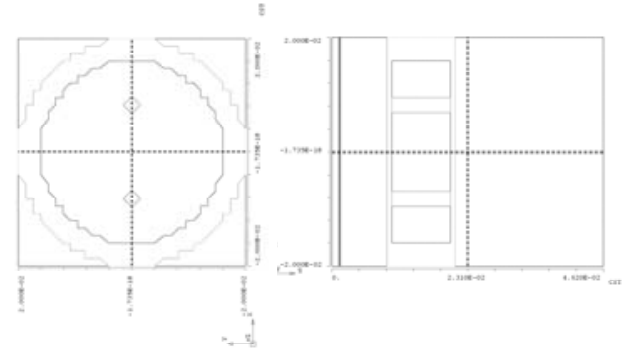

a)

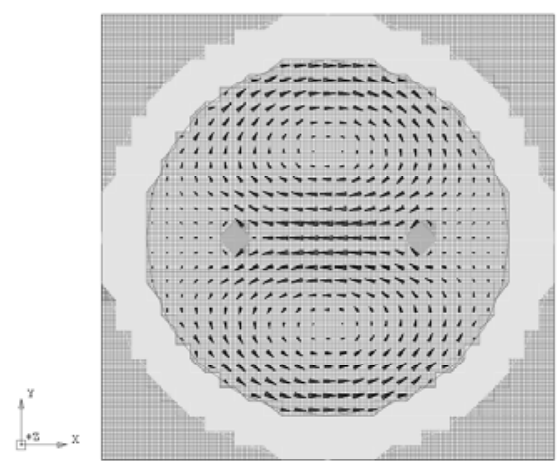

b)

Figure 4 Geometry of the cavity deflector (a) and the magnetic field pattern in the cavity (b)

cavity. As the preliminary simulation, the beam pipe is not included and for normal conducting cavity, only small radius beam pipe required so the TE11 mode in the beam pipe is neglected here.

Unlike the deflection in the meander plane, the cavity deflector use magnetic field to kick the beam. The force is proportional to the velocity of the beam. The impulse on a bunch is proportional to $e B \omega L$, and has nothing to do with the velocity of the bunch. That means the electron can be accelerated to even higher energy and still get effective kicked. The effective length of the cavity is only about half wavelength, because all the full periods are integral to zero. And the effective length is inverse proportional to frequency. The center bunch is kicked up and down and the integral kick is back to zero. The cavity can be run in CW mode, and the RF signal of the cavity can be synchronize with accelerator RF so as to bypass the problem of the photoconductive switch jitter.

The power requirement of the cavity is proportional to $\omega^{-\frac{3}{2}}$. X band is selected to reduce power requirement for an equaling impulse.
For two bunches separation with $500 \mathrm{fs}$, the image and related histogram is shown in figure 5. In this run, $104 \mathrm{~W}$ $\mathrm{RF}$ power is fed into the deflector. And the impulse difference between the two bunches is comparable with the meander plane type used in the current streak camera.
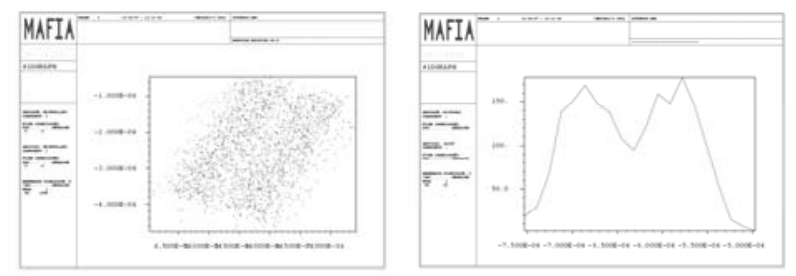

Figure 5 Image of two bunches with 500fs separation (left) and its histogram (right)

That means the difference shown on the final image and histogram is comparable with the current streak camera.

\section{CONCLUSION}

We built an end-to-end model of the ALS streak camera in MAFIA. The model start from the electron bunches on cathode and ended at the image monitor. And the model included the acceleration field, the solenoid focus, the meander bias field and also the dynamic sweep field. The space charge is not included in the model. The agreement between the simulations and experiments of the ALSSC prove the availability of the model. We use the model analyses the interaction between the electrons bunches and the surrounding EM fields and discovered several intrinsic limitation of the meander line based deflector. A new cavity based deflector is proposed and preliminary simulation shows the only $\sim 100 \mathrm{~W} X$ band RF power is required to get comparable deflection effect.

\section{REFERENCES}

[1] http://www.hamamatsu.com

[2] M. M. Shakya and Zenghu Chang, "An accumulative x-ray streak camera with 280-fs resolution" Proceedings of SPIE, Vol. 5534, p125.

[3] G. Naylor and K. Scheidt, "A sub-picosecond accumulating streak camera for X-rays" Meas. Sci. Technol. 12(2001) 1858

[4] J. Feng, W. Wan et al, "An ultra-fast x-ray streak camera for the study of magnetization dynamics" Preceding of SPIE Vol. $5920 \mathrm{p}$

[5] W. Wan, "Study of the aberrations of the magnetic lens of the picosecond streak camera at the ALS" internal tech note

[6] G. Huang J. M. Byrd et al, "Modeling of ultrafast streak camera" EPAC'06, Edinburgh, p1250, http://www.jacow.org

[7] K. Akai et al, "Crab cavity for the B-Factories", Int. J. Mod. Phys. A 2B 1(1993) 757.

[8] "Crab cavity summary", http://ilc-dms.fnal.gov/

[9] http://www.paradisedata.com/ 